\title{
Lithium Intercalation into Opened Single-Wall Carbon Nanotubes: Storage Capacity and Electronic Properties
}

\author{
H. Shimoda, ${ }^{1}$ B. Gao,${ }^{2}$ X. P. Tang, ${ }^{1}$ A. Kleinhammes, ${ }^{1}$ L. Fleming, ${ }^{1}$ Y. Wu, ${ }^{1,2, *}$ and O. Zhou ${ }^{1,2, \dagger}$ \\ ${ }^{1}$ Department of Physics and Astronomy, University of North Carolina, Chapel Hill, North Carolina 27599 \\ ${ }^{2}$ Curriculum in Applied and Materials Sciences, University of North Carolina, Chapel Hill, North Carolina 27599
}

(Received 15 August 2001; published 14 December 2001)

\begin{abstract}
The effects of structure and morphology on lithium storage in single-wall carbon nanotube (SWNT) bundles were studied by electrochemistry and nuclear magnetic resonance techniques. SWNTs were chemically etched to variable lengths and were intercalated with $\mathrm{Li}$. The reversible Li storage capacity increased from $\mathrm{LiC}_{6}$ in close-end $\mathrm{SWNT}$ s to $\mathrm{LiC}_{3}$ after etching, which is twice the value observed in intercalated graphite. All the nanotubes became metallic upon intercalation of $\mathrm{Li}$, with the density of states at the Fermi level increasing with increasing Li concentration. The enhanced capacity is attributed to Li diffusion into the interior of the SWNTs through the opened ends and sidewall defects.
\end{abstract}

DOI: 10.1103/PhysRevLett.88.015502

Recent studies have shown that carbon nanotubes [1,2] have promising materials properties for a variety of technological applications [3]. Both multiwall [4] and single-wall carbon nanotubes (SWNTs) $[5,6]$ are shown to undergo reversible redox reaction with electron donors such as alkali metals resulting in a significantly reduced electrical resistivity [5,7] and electronic work function [8]. The electrochemical reactivity and the porosity make nanotubes, especially SWNT bundles, attractive host materials for energy storage. Molecular dynamic simulation $[9,10]$ predicted that the alkali metal storage capacity of SWNT bundles is substantially higher than that of the intercalated graphite and disordered carbon, the current anode materials in rechargeable Li-ion batteries [11,12]. The closed and relatively defect-free structure of the SWNTs, however, makes the interior space inaccessible for intercalation without processing. Experimentally, the reversible capacity was found to depend on the quality and morphology of the SWNT containing materials. The reported values vary in the range of $\mathrm{Li}_{1-1.6} \mathrm{C}_{6}[13,14]$. The intercalants reside in the interstitial sites of the SWNT bundles $[15,16]$.

Here we show by electrochemical and nuclear magnetic resonance (NMR) techniques that the specific lithium capacity increased from $\mathrm{LiC}_{6}$ to $\mathrm{LiC}_{3}$ when the SWNTs were chemically etched to short segments. All the SWNTs become metallic upon $\mathrm{Li}$ intercalation, with the electronic density of states at the Fermi level, $g\left(E_{F}\right)$, increasing with increasing Li concentration, from 0.022 states/(eV-spinatom) in pristine SWNTs to 0.043 states/(eV-spin-atom) in $\mathrm{LiC}_{3}$. The observed $\mathrm{Li} / \mathrm{C}$ ratio is comparable to the theoretical value [10] and is attributed to $\mathrm{Li}$ diffusion into the individual SWNTs.

SWNT bundles used in this study were synthesized by the laser ablation method [17] under the conditions described elsewhere [18]. All the samples were $10 \%{ }^{13} \mathrm{C}$ isotope enriched from $1.1 \%$ of the natural abundance. As-synthesized materials were purified by first reflux in $\mathrm{H}_{2} \mathrm{O}_{2}$ solution then by filtration. X-ray diffraction and
PACS numbers: 61.46.+w

transmission electron microscopy (TEM) measurements indicated that the purified samples contain over $90 \%$ SWNT bundles over $10 \mu \mathrm{m}$ in length and $30-50 \mathrm{~nm}$ in bundle diameter. The average SWNT diameter was estimated to be $1.4 \mathrm{~nm}$ by $\mathrm{x}$-ray and Raman measurements. The purified SWNTs were processed to shorter bundles by sonication in 3:1 ratio of $\mathrm{H}_{2} \mathrm{SO}_{4}$ and $\mathrm{HNO}_{3}$ solution [19] for 10-24 h. All the samples were annealed at $500^{\circ} \mathrm{C}$ for $1 \mathrm{~h}$ at $5 \times 10^{-6}$ torr vacuum before use. TEM examinations showed that the averaged lengths of the SWNT bundles were reduced to $\sim 4$ and $\sim 0.5 \mu \mathrm{m}$ after 10 and $24 \mathrm{~h}$ of processing, respectively (Fig. 1).

The samples were electrochemically reacted with lithium using a two-electrode cell with Li foil and SWNTs deposited on a stainless steel plate as the two working electrodes [18]. The amount of $\mathrm{Li}$ intercalated into the SWNTs was calculated from the time and the current used. The second-cycle cell voltage versus $\mathrm{Li} / \mathrm{C}$ ratio data obtained from the as-purified SWNTs (A) and two etched SWNT samples with the average bundle length of $4 \mu \mathrm{m}(\mathrm{B})$ and $0.5 \mu \mathrm{m}(\mathrm{C})$ are shown in Fig. 2. Similar to the previous reports $[13,14]$, the as-purified SWNT A was reversibly intercalated to a saturation ratio of $\mathrm{LiC}_{6}$. No distinct redox peaks were observed in intercalation and deintercalation reactions from the derivative plot $(d X / d V$ vs $X, X$ is the lithium concentration expressed as $\mathrm{Li}_{x} \mathrm{C}_{6}$ ) of the same data (Fig. 2a, inset). This is consistent with continuous and random filling of the SWNT bundle interstitial sites by the Li ions, as opposed to the staging phenomena observed in graphite [14].

For the chemically etched samples, the reversible capacity increased to $\mathrm{LiC}_{3}$ and $\mathrm{LiC}_{2.8}$ for samples $\mathrm{B}$ and $\mathrm{C}$, respectively (Figs. $2 b$ and $2 c$ ). These are twice the value observed in lithium intercalated graphite and as-purified SWNTs. It is worthwhile to note that despite the factor of 10 differences in length, samples B and C have essentially the same capacity. Clear redox peaks appeared at $\sim 0.9$ and $1.5 \mathrm{~V}$ on $\mathrm{Li}$ intercalation, and 2.0 and $1.5 \mathrm{~V}$ on deintercalation. These peaks are more pronounced in 


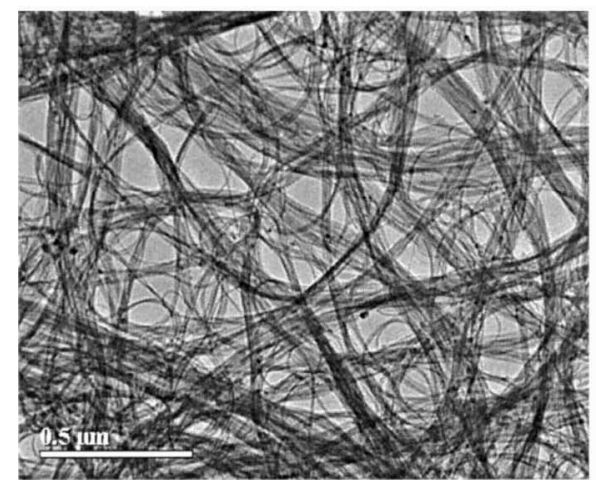

(A)

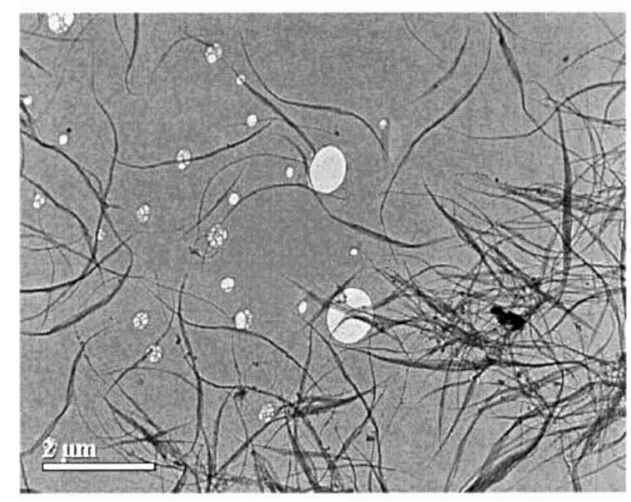

(B)

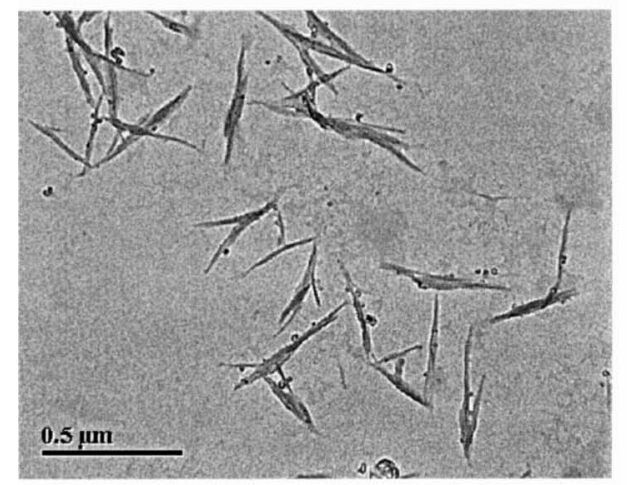

(C)

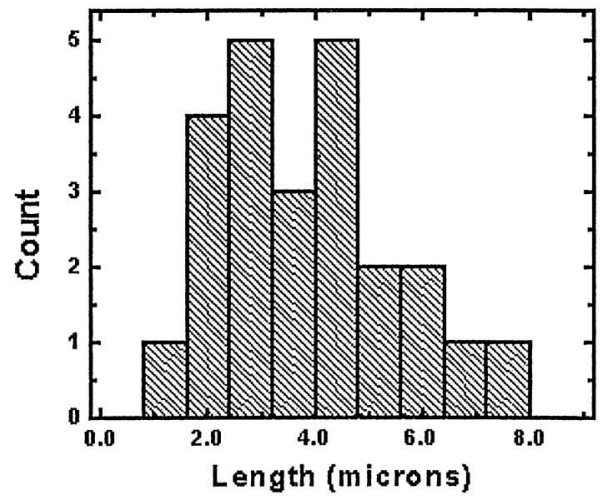

(D)

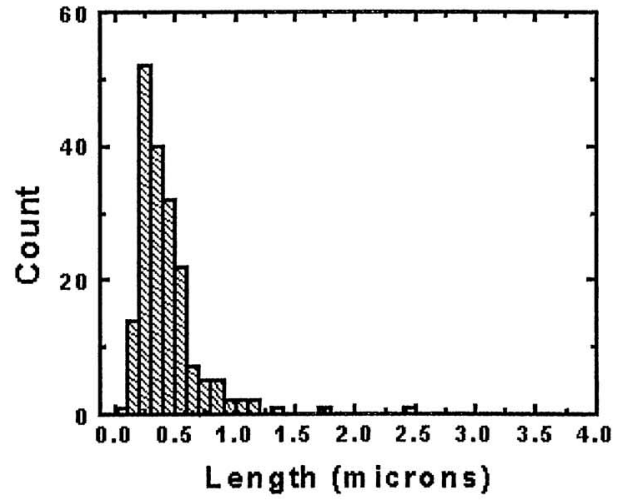

(E)

FIG. 1. Transmission electron microscopy images of (A) as-purified SWNTs, and SWNTs after chemically etched for $10 \mathrm{~h}$ (B) and $24 \mathrm{~h}(\mathrm{C})$ in strong acid. The histograms (D) and (E) show that the length distribution of sample (B) is centered at $4 \mu \mathrm{m}$ and (C) at $0.3-0.5 \mu \mathrm{m}$ and the length distribution of sample (C) is much narrower than that of sample (B).

sample C, which has a shorter average bundle length and a much narrower length distribution. The two peaks are not due to $\mathrm{Li}$ reaction with $\mathrm{H}$ containing functional groups on SWNTs since proton NMR measurement showed that the amount of $\mathrm{H}$ in the etched samples is less than 0.2 at. \%. It is not likely that the amorphous carbon $(\alpha-\mathrm{C})$ impurity phase contributes to these two redox reactions, because no distinct redox peak was observed in raw SWNT materials which typically contain a higher percentage of $\alpha$-C than the samples investigated here. We attribute the excess Li capacity and distinct redox peaks observed in the etched samples to Li diffusion into the interior space of the cut SWNTs, either through the opened ends or defects on the sidewalls created by chemical etching. The voltage hysteresis, which is related to the kinetics of the electrochemical reaction, decreased by $\sim 0.5 \mathrm{~V}$ in sample $\mathrm{C}$ compared with sample A. This indicates that a shorter diffusion path or/and a lower diffusion barrier in the etched samples which is consistent with the observation of smaller bundle diameter and length in sample $\mathrm{C}$.

The chemical composition and electronic properties of Li intercalated SWNTs were also investigated using ${ }^{13} \mathrm{C}$ 


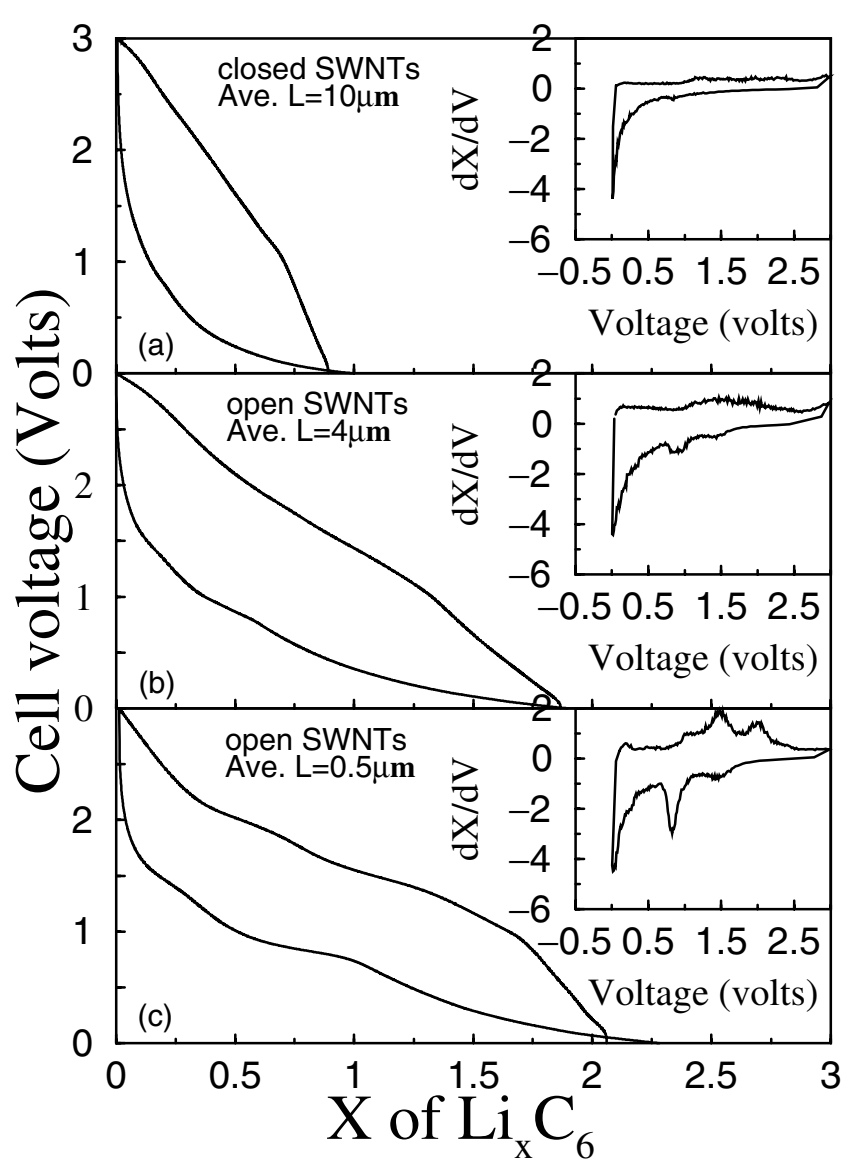

FIG. 2. Second cycle intercalation (discharge) and deintercalation (charge) data collected from the as-purified SWNTs (a) and etched SWNTs with the average bundle length of $4 \mu \mathrm{m}$ (b) and $0.3-0.5 \mu \mathrm{m}$ (c). The data were collected using a twoelectrode cell with Li foil and SWNT film as the two electrodes. A $1 \mathrm{M}$ solution of $\mathrm{LiClO}_{4}$ in 1:1 volume ratio of ethylene carbonate and dimethyl carbonate was used as the electrolyte. Detailed experimental conditions were described elsewhere [13]. Lithium intercalation and deintercalation were carried out using the galvanostatic mode at $50 \mathrm{~mA} / \mathrm{g}$ current between $0-3 \mathrm{~V}$.

and ${ }^{7} \mathrm{Li}$ NMR techniques. Samples were prepared by mixing of $\mathrm{LiBH}_{4}$ and SWNTs at the Li:C molar ratio of 1:6 and $1: 3$,

$$
\begin{aligned}
x \mathrm{LiBH}_{4}+\mathrm{C}(\mathrm{SWNT}) \rightarrow & \mathrm{LI}_{x} \mathrm{C}(\mathrm{SWNT}) \\
& +(x / 2) \mathrm{B}_{2} \mathrm{H}_{6}+(x / 2) \mathrm{H}_{2}
\end{aligned}
$$

[20]. SWNT samples A (as-purified) and C (etched, with the average bundle length of $0.5 \mu \mathrm{m}$ ) from the same batches as the materials investigated by electrochemistry were used.

For sample A, the composition of the Li-SWNT phase was determined to be $\mathrm{LiC}_{5.7}$ and $\mathrm{LiC}_{5.5 \pm 0.7}$ when the molar ratio of $\mathrm{Li}$ and $\mathrm{C}$ (SWNT) used in the reaction was 1:6 and 1:3, respectively (Table I). This indicates unambiguously that there is a distinct saturation phase with the $\mathrm{Li} / \mathrm{C}$ ratio of $\mathrm{LiC}_{6}$ for SWNTs with an enclosed structure. For sample $\mathrm{C}$, the composition of the Li-SWNT phase is determined to be $\mathrm{LiC}_{3.2}$ when the $\mathrm{Li}: \mathrm{C}$ molar ratio used in the reaction is 1:3, twice the value observed in sample $\mathrm{A}$ (Table I). The NMR results from samples made by the solid state reactions are consistent with the saturation $\mathrm{Li} / \mathrm{C}$ ratios obtained from the electrochemical measurements for the as-purified and the etched SWNTs.

Figure 3 displays the ${ }^{13} \mathrm{C}$ nuclear spin-lattice relaxation (NSLR) curves of the SWNTs before and after Li intercalation measured at room temperature and at $9.4 \mathrm{~T}$. As previously reported [18], the saturation recovery curve $M^{*}(t)=1-M(t) / M$ (infinite) ( $M$ is the magnetization of ${ }^{13} \mathrm{C}$ nuclei) of the pristine SWNT sample can be fit very well with a double-exponential function:

$$
M^{*}(t)=\alpha \exp \left(-t / T_{1 \alpha}\right)+(1-\alpha) \exp \left(-t / T_{1 \beta}\right)
$$

with fitting parameters $a=0.33+0.07, T_{1 a}=12.0+$ $0.5 \mathrm{~s}$, and $T_{1 b}=99+2 \mathrm{~s}$. The fast-relaxing component ( $33 \%$ by mass) is attributed to the metallic SWNTs with an estimated $g\left(E_{F}\right)$ of 0.022 states/(eV-spin-atom) while the slow-relaxing component (67\% by mass) is attributed to the semiconducting SWNTs [18]. As shown in Fig. 3, the ${ }^{13} \mathrm{C}$ NSLR curves of the $\mathrm{LiC}_{6}$ and $\mathrm{LiC}_{3}$ phases from the SWNT samples $\mathrm{A}$ and $\mathrm{C}$ can be fitted with a single-exponent function with fitted $T_{1}$ of $(6 \pm$ $0.5) \mathrm{s}$ and $(3.2 \pm 0.3) \mathrm{s}$, respectively. Assuming the same hyperfine-coupling constant for SWNTs before and after intercalation, the $g\left(E_{F}\right)$ of the two samples are estimated to be 0.031 and 0.043 states $/(\mathrm{eV}$-spin-atom), respectively (Table I). Figure 3 also shows that the ${ }^{13} \mathrm{C}$ NSLR curves from sample A with the nominal composition of $\mathrm{LiC}_{3}$ and $\mathrm{LiC}_{6}$ are the same. This confirms the Li-SWNT phases in the two samples have the same chemical composition $\left(\mathrm{LiC}_{6}\right)$ despite the different $\mathrm{Li} / \mathrm{C}$ atomic mole ratios used for the reaction.

The factor of 2 increase in the Li uptake in the etched SWNTs is attributed to Li filling of the interior space of the SWNTs, in addition to the interstitial sites within the bundles. In contrast to the as-purified SWNTs with

TABLE I. Saturation Li composition and density of states of different SWNT materials. Note: The $g\left(E_{F}\right)$ of metallic pristine SWNT is 0.022 states/(eV-spin-atom) [18].

\begin{tabular}{lcc}
\hline \hline Starting material & Purified SWNTs & Etched SWNTs \\
\hline Saturation $\mathrm{Li} / \mathrm{C}$ ratio & $\mathrm{LiC}_{5.7}$ & $\mathrm{LiC}_{3.2}$ \\
Density of states at the Fermi level & 0.031 & 0.043 \\
[states/(eV-spin-atom)] & & \\
\hline \hline
\end{tabular}




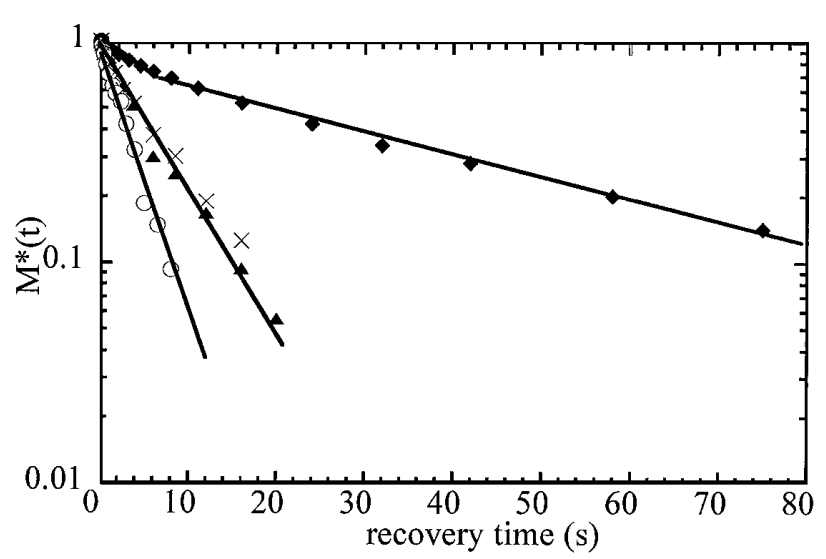

FIG. 3. ${ }^{13} \mathrm{C}$ nuclear spin-lattice relaxation (NSLR) curves measured at room temperature and at $9.4 \mathrm{~T}$. The curve from the pristine sample (filled diamond) can be fitted with a doubleexponential function with the respective relaxation time of 12 and $99 \mathrm{~s}$. The fast-relaxing component $(33 \mathrm{wt} . \%)$ is attributed to the metallic SWNTs while the slow-relaxing component is attributed to the semiconducting SWNTs. The NSLR curves of the $\mathrm{LiC}_{6}$ (as-purified $\mathrm{SWNT}$, filled triangle) and $\mathrm{LiC}_{3}$ (etched SWNT, open circle) samples can be fitted with a singleexponential function with the respective $T_{1}$ of 6.5 and $3.2 \mathrm{~s}$, respectively. The nominal $\mathrm{LiC}_{3}$ (as-purified SWNT, cross) has the same NSLR curve as the $\mathrm{LiC}_{6}$ (as-purified SWNT, filled triangle) sample with a $T_{1}$ of $6.5 \mathrm{~s}$.

closed ends and large aspect ratios, Li can diffuse into the etched tubes via the open ends and possibly sidewall defect sites created by oxidation. The high Li uptake is consistent with our previous observation of enhanced $\mathrm{Li} / \mathrm{C}$ ratio in ball-milled SWNTs where the nanotubes were fractured [21].

We thank J. Liu for providing the initial etched nanotube samples, L. McNeil and J. P. Lu for helpful discussions and for technical assistance. This work was partially supported by the Office of Naval Research through a MURI program at UNC and the National Science Foundation.

*Email address: yuewu@ physics.unc.edu

†Email address: zhou@physics.unc.edu

[1] S. Iijima, Nature (London) 354, 56-58 (1991).

[2] M.S. Dresselhaus, G. Dresselhaus, and P.C. Eklund, Science of Fullerenes and Carbon Nanotubes (Academic Press, New York, 1996).

[3] P. M. Ajayan and O. Zhou, in Carbon Nanotubes: Synthesis, Structure, Properties, and Applications, Topics in Applied Physics Vol. 80, edited by M.S. Dresselhaus,
G. Dresselhaus, P. Avouris (Springer-Verlag, Heidelberg, 2000).

[4] O. Zhou, R. M. Fleming, D. W. Murphy, C. T. Chen, R. C. Haddon, A. P. Ramirez, and S.H. Glarum, Science 263, 1744-1747 (1994).

[5] R. S. Lee, H. J. Kim, J. E. Fischer, A. Thess, and R. E. Smalley, Nature (London) 388, 255-257 (1997).

[6] A. M. Rao, P. C. Eklund, S. Bandow, A. Thess, and R. E. Smalley, Nature (London) 388, 257-259 (1997).

[7] L. Grigorian, K. A. Williams, S. Fang, G. U. Sumanasekera, A. L. Loper, E. C. Dickey, S. J. Pennycook, and P. C. Eklund, Phys. Rev. Lett. 80, 5560-5563 (1998).

[8] S. Suzuki, C. Bower, Y. Watanabe, and O. Zhou, Appl. Phys. Lett. 76, 4007-4009 (2000).

[9] G. Gao, T. Cagin, and W. A. Goddard, Phys. Rev. Lett. 80, 5556-5559 (1998).

[10] J. Zhao, A. Buldum, J. Han, and J. P. Lu, Phys. Rev. Lett. 85, 1706-1709 (2000).

[11] Lithium Batteries: New Materials, Developments and Perspectives, edited by G. Pistoia, Industrial Chemistry Library, Vol. 5 (Elsevier, Amsterdam, 1994).

[12] J. R. Dahn, T. Zhang, Y. Liu, and J. S. Xue, Science 270, 590-593 (1995).

[13] B. Gao, A. Kleinhammes, X. P. Tang, C. Bower, L. Fleming, Y. Wu, and O. Zhou, Chem. Phys. Lett. 307, 153 (1999).

[14] A. Claye, J. E. Fischer, C. B. Huffmand, A. G. Rinzler, and R. E. Smalley, J. Electrochem. Soc. 147, 2845-2852 (2000); A. Claye, S. Rahman, J.E. Fischer, A. Sirenko, G. U. Sumanasekera, and P. C. Eklund, Chem. Phys. Lett. 333, 16-22 (2001).

[15] C. Bower, A. Kleinhammes, Y. Wu, and O. Zhou, Chem. Phys. Lett. 288, 481-486 (1998); S. Suzuki, C. Bower, and O. Zhou, Chem. Phys. Lett. 285, 230-234 (1998).

[16] A. Claye, J. E. Fischer, and A. Metrot, Chem. Phys. Lett. 330, 61-67 (2000); A. S. Claye, N. M. Nemes, A. Janossy, and J. E. Fischer, Phys. Rev. B 62, R4845-4848 (2000).

[17] A. Thess, R. Lee, P. Nikdaev, H. Dai, P. Petit, J. Robert, C. Xu, Y. H. Lee, S. G. Kim, A. G. Rinzler, D. T. Colbert, G. E. Scuseria, D. Tomanek, J.E. Fischer, and R.E. Smalley, Science 273, 483-487 (1996).

[18] X. P. Tang, A. Kleinhammes, H. Shimoda, L. Fleming, C. Bower, S. Sinha, O. Zhou, and Y. Wu, Science 228, 492 (2000).

[19] J. Liu, A. Rinzler, H. Dai, J. Hafner, A. R. Bradley, P. Boul, A. Lu, T. Iverson, A. K. Shelimov, C. Huffman, F. Rodriguez-Macias, Y. Shon, R. Lee, D. Colbert, and R. E. Smalley, Science 280, 1253-1256 (1998).

[20] D. W. Murphy, M. J. Rosseinsky, R. M. Fleming, R. Tycko, A. P. Ramirez, R. C. Haddon, T. Siegrist, G. Dabbagh, J. C. Tully, and R. E. Walstedt, J. Phys. Chem. Solids 53, 1321-1332 (1992).

[21] B. Gao, C. Bower, J. Lorentze, L. Fleming, A. Kleinhamme, X. P. Tang, L. E. McNeil, Y. Wu, and O. Zhou, Chem. Phys. Lett. 327, 69-75 (2000). 